\title{
DISFUNCIÓN MICCIONAL POST-BRAQUITERAPIA EN EL CÁNCER PROSTÁTICO
}

\author{
Salvador Arlandis Guzmán, Miguel Angel Bonillo García y Enrique Broseta Rico.
}

Unidad de Tracto Urinario Inferior. Servicio de Neurología y Urodinámica. Servicio de Urología. Hospital Universitario La Fe. Valencia. España.

\begin{abstract}
Resumen.- La aparición de síntomas urinarios postbraquiterapia es un hecho muy frecuente, que alcanza su máximo entre el primer y tercer mes postimplante, normalizándose alrededor del año. La incidencia de retención urinaria postimplante oscila entre el 1.5 y el $27 \%$. Un porcentaje bajo de pacientes (0-8.7\%) necesitará una cirugía desobstructiva para resolver una retención urinaria prolongada o síntomas obstructivos severos. Los alfabloqueantes pueden ser útiles para aliviar los sintomas urinarios postimplante, aunque no reducen la necesidad de cirugía posterior. Existen varios factores clínicos predictivos de aparición de complicaciones urinarias. Un paciente con puntuación IPSS preimplante alta, próstata de gran tamaño, con signos funcionales
\end{abstract}

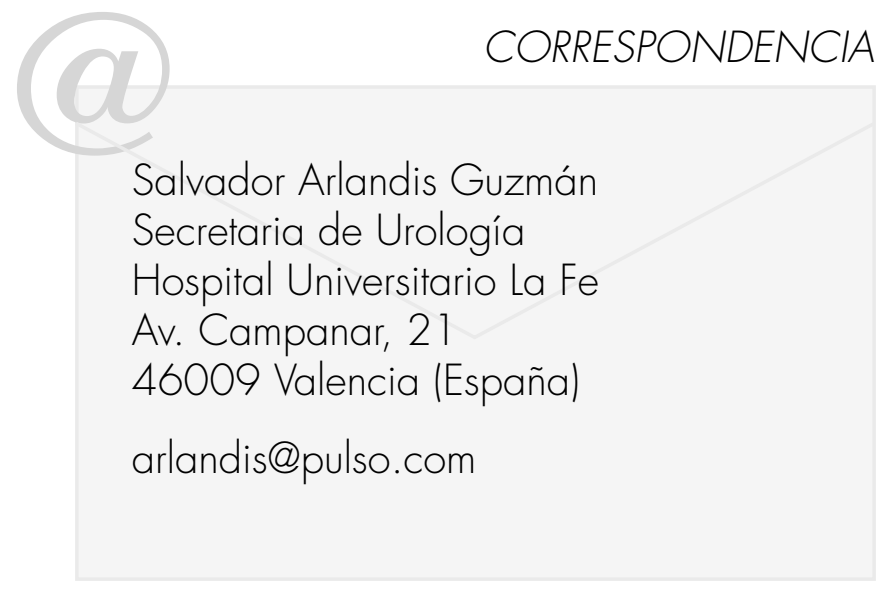

obstructivos $(Q \max$ bajo, residuo alto y parámetros urodinámicos de obstrucción), tiene alto riesgo de presentar retención urinaria o síntomas urinarios prolongados. Esto repercutirá claramente en la calidad de vida del paciente, lo que lo hace candidato a otro tipo de terapias. El empleo de una técnica de implantación periférica (que asegure bajas dosis en uretra) y evitar el plano uretral en la inserción de las agujas puede minimizar el daño uretral asociado a la aparición de complicaciones urinarias. La incontinencia postbraquiterapia es una complicación poco frecuente, pero que aumenta de forma dramática en pacientes con RTUP previa o que precisan de la misma para resolver una retención urinaria mantenida. Es fundamental conocer esta circunstancia para informar debidamente al paciente y planificar una RTUP lo más cuidadosa posible.

Palabras clave: Braquiterapia prostática. Complicaciones urinarias. Incontinencia urinaria. Retención urinaria. Cáncer de próstata.

Summary.- OBIECTIVES: The development of urinary symptoms after brachytherapy is very frequent, reaching a maximum between the first and third month after the implant and returning back to normal around one year. The incidence of acute urinary retention varies between 1.5 and $27 \%$. A low percentage of patients (0-8.7\%) will need surgery to solve an extended urinary retention or severe obstructive voiding symptoms. Alphablockers may be useful to alleviate voiding symptoms after the implant, although they do not reduce the need for surgery.

There are several predictive clinical factors for development of urinary complications. A patient with high IPPS score before implant, large size prostate, obstructive functional signs (Low Q max, high postvoid residual, and obstruction urodynamic parameters) has a high risk 
for urinary retention or extended urinary symptoms. This has repercussion on the quality of life of the patient, making them candidates to other therapies. The performance of a peripheral implant technique lguaranteeing low doses at urethra) and avoiding the urethral plane when inserting the needles may minimize urethral damage associated with development of urinary complications. Urinary incontinence after brachytherapy is a rare complication, but its frequency increases dramatically after transurethral resection of the prostate or requiring it to solve a sustained urinary retention. It is fundamental to know this circumstance to inform the patient adequately and to plan the most careful TURP.

Keywords: Prostatic brachytherapy. Urinary complications. Urinary incontinence. Urinary retention. Prostate cancer.

\section{INTRODUCCIÓN}

La braquiterapia prostática implica la colocación de fuentes radiactivas en la glándula prostática. Dado su corto rango de irradiación emitido, altas dosis de energía pueden ser liberadas por estas fuentes dentro de la próstata, evitando una excesiva irradiación a órganos vecinos. El desarrollo de nuevas tecnologías ha permitido mejorar progresivamente no sólo la colocación de las semillas radiactivas, sino también la distribución de la dosis de irradiación. Como resultado de todo ello, la braquiterapia prostática transperineal con implante permanente constituye una opción definitiva de tratamiento en pacientes con un cáncer de próstata clínicamente localizado. Aunque no disponemos de trabajos prospectivos controlados y aleatorizados, las tasas de control bioquímico en pacientes tratados mediante esta técnica son similares a aquellos tratados mediante radioterapia externa o prostatectomía radical (1).

En los últimos años esta modalidad de tratamiento ha experimentado un auge muy significativo, tanto por su eficacia comparada con la cirugía, como por su rapidez, reproducibilidad, escasa invasividad y relativa baja tasa de complicaciones. Por otro lado, aunque las semillas radioactivas son caras, la infraestructura necesaria para efectuar el implante es relativamente económica y sencilla (un ecógrafo y un sistema informático acoplado al mismo).

Sin embargo, a pesar de su carácter mínimamente invasivo y de ser una técnica generalmente bien tolerada, la braquiterapia prostática no está exenta de efectos secundarios digestivos, urinarios y disfunción eréctil.
La incidencia de complicaciones digestivas es baja $(2 \%)$, consistiendo principalmente en proctitis (2), y su presentación es más tardía que la sintomatología urinaria. Por otro lado, entre el 6 y el $53 \%$ de los pacientes sometidos a braquiterapia pueden desarrollar una disfunción eréctil después del implante (3). El mecanismo por el que se produce una disfunción eréctil post-implante es controvertido. El deterioro inicial de la función eréctil debe ser atribuido al dolor y al disconfort eyaculatorio, al ser el efecto de la braquiterapia gradual con el tiempo, a diferencia de la prostatectomía radical, donde el efecto es inmediato, o en la radioterapia externa, donde la función eréctil se deteriora entre los 12 y 24 meses post-radiación $(3,4)$.

Pero los efectos secundarios más frecuentes son los urinarios, y la mayoría de los pacientes los experimentan en algún momento durante el primer año post-implante. El objetivo de este artículo es hacer una revisión de los efectos secundarios urinarios asociados a la braquiterapia por cáncer prostático, su fisiopatología, factores que influyen en su aparición y medidas preventivas o terapéuticas que han de tenerse en cuenta.

\section{SÍNTOMAS DEL TRACTO URINARIO INFERIOR ASOCIADOS A BRAQUITERAPIA: HIPÓTESIS FISIOPATOLÓGICAS}

Tras el implante prostático de semillas radioactivas, la mayoría de los pacientes experimentan una serie de síntomas del tracto urinario inferior (STUI) de llenado y de vaciado que afectan en diverso grado su calidad de vida. Polaquiuria y nocturia, urgencia e incluso incontinencia de urgencia, chorro débil, disuria, interrupción miccional y sensación de residuo postmiccional son síntomas que a menudo aparecen tras el tratamiento. Incluso algunos pacientes pueden desembocar en retención aguda de orina que requiera cateterismo vesical transitorio. La evolución cronológica de esta sintomatología muestra un inicio a los pocos días o semanas del implante, alcanzando un pico máximo entre el primer y tercer mes y normalizándose la clínica progresivamente con los meses, alcanzando el nivel sintomático preimplante alrededor del año $(2,5)$.

Los síntomas de llenado como la frecuencia urinaria elevada, y la urgencia pueden tener diferente fisiopatología, y las causas exactas no se conocen con detalle. Una de las hipótesis más plausibles es el daño uretral, tanto mecánico como por irradiación. Existen dos métodos de implantar las semillas en la próstata: el sistema de carga uniforme (tipo Quimby, que sitúa las semillas de forma uniforme por toda la 
glándula) (6) y el sistema de carga periférica (tipo Paterson y Parker, que tiende a localizar las semillas en la zona más externa) (7) (Figura 1). En el primer sistema, aunque la distribución es homogénea y simétrica, la dosis de radiación que recibe la región central donde se sitúa la uretra es mucho mayor que la región periférica. Esto aumenta el riesgo de efectos secundarios como necrosis y fibrosis uretrales, a la vez que es menor la dosis que recibe la zona periférica (donde con más frecuencia se localiza el foco neoplásico). Por lo tanto, la forma más frecuente de sembrar las semillas es utilizando la carga periférica, minimizando de este modo la sobreirradiación uretral $(8,9)$. Además, se intenta evitar el plano vertical inmediatamente por encima de la uretra, minimizando el riesgo de traumatismo uretral directo con el paso de las agujas de siembra de semillas. Sin embargo, aunque la hipótesis del daño uretral es lógica, los trabajos publicados en la literatura son contradictorios en evidenciar la correlación entre dosis uretral y síntomas urinarios, existiendo datos a favor $(10,11)$ y en contra (12-14) de esta hipótesis.

La relación de la sintomatología urinaria con la dosis recibida por la próstata permanece controvertida. Así Desai et al. (15) relacionaron la elevación del IPSS con la actividad total implantada (V80/90/95/100 y D70/90/100/150). Sin embargo, Merrick et al. (16) no encontraron correlación entre el IPSS y la dosis uretral o las dosis totales prostáticas aplicadas. Igualmente, Wallner et al. (9) relacionaron la toxicidad urinaria con una máxima dosis uretral mayor al $250 \%$ de la dosis mínima periférica. Actualmente con los implantes periféricos rara vez la dosis uretral supera el $150 \%$ de la dosis prescrita. Por ello la recomendación es que la dosis uretral esté entre el $100-140 \%$ de la dosis prescrita.

Steggarda et al. (17) observaron una correlación entre la aparición de síntomas urinarios al tercer y sexto mes con la administración de altas dosis en volúmenes prostáticos pequeños $\left(1 \mathrm{~cm}^{3}\right)$ en cuello y base de vejiga. En esta línea se encuentran los resultados del trabajo de Williams et al. (18), que observaron una mayor aparición de síntomas urinarios en pacientes con semillas colocadas más cranealmente (base vesical). Esto se podría explicar por una irritación mecánica o actínica de la zona sensible del cuello y trígono vesical.

Otros muchos factores se han postulado como asociados a un mayor riesgo de aparición de síntomas urinarios tras braquiterapia prostática, pero ninguno ha alcanzado una evidencia clara sobre los demás. El factor más coincidente en la mayoría de autores es el volumen prostático (12-14, 17, 19, 20), pero parece más relacionado con la tendencia a la aparición de retención aguda de orina que a la de síntomas urinarios. Otros factores postulados son contradictorios, y así algunos autores han encontrado correlación con la dosis prostática $(20,21)$, el número de agujas empleadas (21), la edad (17, 21), radioterapia externa añadida (21-23), volumen de zona transicional $(14,24)$ o tipo de radionúclido (25). Con respecto a este último factor, existen dos tipos de radionúclidos utilizados en braquiterapia prostática: el I-125 y el Pd-103. En Europa se utiliza preferentemente el 1-125, siendo pocos los centros que utilizan el Pd-103. La principal diferencia entre ambos isótopos es su vida media, 60 días para el I-125 y 17 para el $\mathrm{Pd}-103$, lo que significa que la dosis total llega a los tejidos más rápidamente con el Pd-103. Es de esperar una más rápida aparición de síntomas urinarias con el $\mathrm{Pd}-103$, así como también una más pronta resolución. En congruencia con esta hipótesis están los trabajos de Allen et al. (25) y WaIlner et al. (1 1), pero otros autores no confirmaron estos hallazgos. Los síntomas iniciales pueden atribuirse al traumatismo mecánico de las agujas, que puede superponerse al efecto de la irradiación precoz del radionúclido, con lo que no serían detectables diferencias clínicas entre los dos tipos de isótopos.

La mayoría de los autores coinciden en afirmar que una presencia de STUI severos antes de la braquiterapia prostática (constatada con una puntuación basal IPSS alta) anuncia la aparición de síntomas urinarios postbraquiterapia, lo que parece francamente lógico $(12,13,17,22,23,25)$. Curiosamente y de forma contraria a lo que pudiera esperarse, Williams et al. (18) observan que aquellos pacientes con peores puntuaciones IPSS prebraquiterapia empeoran menos que los que presentan

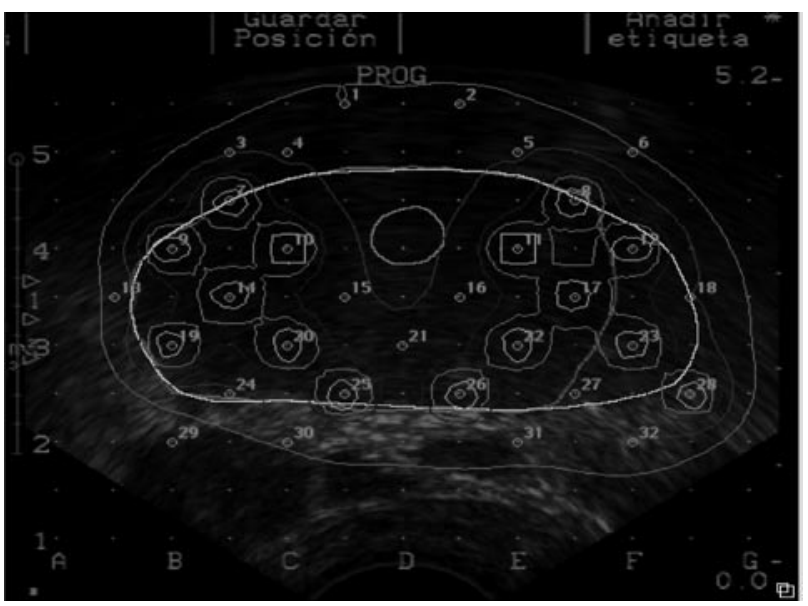

FIGURA 1. Distribución periférica de las semillas. 
puntuaciones más bajas (probablemente, el margen para empeorar es menor, y a fin de cuentas, el IPSS es un cuestionario de medición de síntomas subjetivo autoadministrado).

\section{HALLAZGOS URODINÁMICOS TRAS BRAQUI- TERAPIA PROSTÁTICA}

Pocos autores han estudiado los efectos urodinámicos de la braquiterapia prostática, más allá de una constatación de la aparición de síntomas urinarios y necesidad de catéter vesical por retención aguda de orina.

En un estudio sobre 47 pacientes con STUI postbraquiterapia prostática, Blaivas et al. (26) comparan los hallazgos urodinámicos de este grupo con otro grupo de 541 pacientes generales con STUI no secundarios a braquiterapia. Observa que 37 pacientes $(79 \%)$ refieren síntomas de vejiga hiperactiva, $31(71 \%)$ incontinencia y 21 síntomas de vaciado $(44 \%)$. Al comparar los pacientes con STUI no sometidos a braquiterapia con el grupo postbraquiterapia, observa una incidencia de hiperactividad del detrusor en 252 de 541 pacientes (47\%) en el primer grupo frente a 28 de $33(85 \%)$ del segundo $(p<0.001)$, y de obstrucción uretral en 374 de $541(69 \%)$ vs 24 de $33(73 \%)$ del grupo de braquiterapia $(p=0.85)$. A pesar de no encontrar diferencias con respecto a la presencia de obstrucción, sí constatan mayor incidencia en el grupo de braquiterapia de obstrucción por esclerosis severa de uretra prostática y cuello vesical, y estenosis de uretra membranosa (siendo estos hallazgos menores a un $10 \%$ en el primer grupo). Por otro lado, los autores observan una mayor severidad de los STUI en el grupo de braquiterapia.

La mayoría de trabajos evalúan diversos parámetros urodinámicos como factores predictivos de aparición de STUI y retención urinaria postbraquiterapia, factores que comentaremos posteriormente.

\section{MORBILIDAD URINARIA: COMPLICACIONES AGUDAS Y CRÓNICAS}

Están causadas por los efectos agudos de la radiación y por el traumatismo que se produce durante el implante de las semillas. El implante de las semillas puede producir un hematoma perineal subcutáneo, equimosis, edema y sangrado perineal. Si durante la colocación de las agujas se punciona el cuello vesical o la uretra, puede aparecer hematuria e incluso retención aguda de orina (RAO) por coágulo (27). La morbilidad debida a la radiación aguda está en función de la dosis recibida por la ure- tra, la próstata, el recto y los haces neurovasculares. Ésta será mayor en intensidad y frecuencia en los pacientes que reciben braquiterapia combinada con radioterapia externa. Suele manifestarse en el primertercer mes tras el implante y luego va disminuyendo progresivamente a medida que la radioactividad del isótopo se reduce. Como ya se ha comentado, cada isótopo tiene unas características radiobiológicas distintas, lo que puede condicionar el momento de aparición o el pico de intensidad de los síntomas.

\section{COMPLICACIONES URINARIAS AGUDAS}

1. Síndrome miccional obstructivo y retención urinaria: Suelen aparecer en el $50 \%$ de los pacientes. Se manifiestan principalmente con disuria y debilidad del chorro miccional, lo que ocasiona un deterioro significativo en el flujo medio y máximo de la flujometría libre urinaria y un aumento en el IPSS. Los cambios observados en la medición del residuo post-miccional son menos significativos (5). Los cambios más significativos en el IPSS suelen aparecer en la fase aguda y progresivamente se reducen en 1 ó 2 años hasta alcanzar el nivel previo (15). La RAO ocurre habitualmente al día siguiente del implante y probablemente está relacionada con el edema post-implante y los efectos de los agentes anestésicos, ya que la radiación liberada es todavía insignificante (28). Pese a todo ello, el índice de retenciones urinarias es bajo, entorno al $1.5-27 \%(12,27-31)$, y se suele resolver con la colocación de una sonda vesical por espacio de unas semanas y tratamiento médico con $\alpha$-bloqueantes. Pocos de estos pacientes $(0-8.7 \%)$ requerirán una resección transuretral de próstata (RTUP) desobstructiva por RAO refractaria. El uso de un $\alpha$-bloqueante no reduce el riesgo de retención prolongada (más de 3 días) ni reduce la necesidad de una cirugía desobstructiva postimplante (15).

2. Síndrome miccional irritativo: Es, sin duda, el efecto secundario más frecuente, presente en el $46-66 \%$ de los pacientes $(5,33)$, y se manifiesta como escozor al orinar, frecuencia-urgencia, nocturia, acompañado en ocasiones de hematuria.

La sintomatología miccional secundaria a la radiación recibida sobre vejiga es clasificada por la Radiation Therapy Oncology Group (RTOG) en cinco grados. La toxicidad grado 0 implica la ausencia de cambios y la toxicidad grado 1 indica una ligera atrofia epitelial o una telangiectasia menor, pudiendo haber microhematuria. En la toxicidad grado 2 existe una telangiectasia generalizada, frecuencia miccional moderada y hematuria macroscópica intermitente. En la grado 3 la frecuencia miccional es severa, con escozor intenso al orinar, telangiectasia genera- 
lizada severa (a menudo con petequias), hematuria frecuente, y puede existir reducción de la capacidad vesical (<150 ml). En la toxicidad grado 4 hay necrosis, cistitis hemorrágica severa y reducción de la capacidad vesical (capacidad < $100 \mathrm{ml}$ ). Esta escala es una valoración subjetiva y no está universalmente aceptada, aunque probablemente sea la más utilizada en la literatura.

Prada et al. (2) clasifica a los pacientes con esta clínica en tres grupos en función de su comportamiento: un tercio de sus pacientes presentan una clínica insignificante, otro tercio presenta un síndrome irritativo-obstructivo que se resuelve con hábitos higiénico-dietéticos, o bien con la toma de antiinflamatorios o $\alpha$-bloqueantes de forma esporádica, y el otro tercio restante presenta una clínica de mayor intensidad, grado 1-2 de la RTOG, que obliga a tratamiento antiinflamatorio y $\alpha$-bloqueante pautado.

La clínica aparece entre el primer y tercer mes para luego disminuir de forma progresiva, de tal forma que alrededor del $66-92 \%$ de los pacientes tendrán una mejoría de esta clínica con el tiempo hasta ser mínima o indetectable en el $6^{\circ}-12^{\circ}$ mes $(2$, $5,34,35)$. No obstante puede que algunos pacientes experimenten un cuadro de reagudización de la clínica una vez desaparecida la misma, habitualmente de corta duración y menor intensidad, o esta clínica no desaparezca y permanezca presente. Wallner et al. (33), detectó a los dos años del implante que un $14 \%$ de sus pacientes tenían sintomatología urinaria persistente grado $\geq 2$ de la RTOG.

\section{COMPLICACIONES A LARGO PLAZO}

1. Estenosis uretral o del cuello vesical: Uno de los principales factores para el desarrollo de este tipo de complicación es el antecedente de cirugía prostática previa al implante. Ello se debe a que la uretra prostática y el cuello vesical se encuentran muy poco vascularizados después de la cirugía, lo que hace que toleren mal altas dosis de radiación (27). Otro factor relacionado es la dosis que recibe la uretra bulbo-membranosa, de tal forma que una dosis $\leq 50$ $\%$ de la dosis total a dicho nivel, evitaría este tipo de complicación (2). También se ha relacionado con la colocación de las semillas en posición demasiado caudal, justo anterior al ápex prostático. Técnicas de imagen de alta precisión y la colocación de una sonda vesical en el momento del implante ayuda a visualizar mejor la uretra y reducir este tipo de complicación (27).

2. Incontinencia urinaria: Es una de las complicaciones más limitantes. Tiene una incidencia del 0 al 83
$\%$, que aumenta en el caso de pacientes tratados con braquiterapia combinada con radioterapia externa y en aquellos que han sido sometidos a RTU-P.

\section{RETENCIÓN AGUDA DE ORINA}

El síntoma urinario más significativo tras braquiterapia prostática es la obstrucción urinaria que precisa cateterismo. La incidencia varia del 5 al $28 \%$ $(13,18,22-25,29,36-39)$ (Tabla I). La mayoría de las veces es suficiente con unos días de sondaje vesical, siendo prolongado en un porcentaje inferior al $2 \%$ en la mayoría de las series. La presencia de un residuo postmiccional elevado en algunos casos puede aconsejar la práctica de cateterismo limpio intermitente durante algunas semanas para mejorar los síntomas de los pacientes (38). Algunos autores como Crook et al. han referido la necesidad de sondaje vesical durante periodos largos de tiempo (3-18 meses) (29).

La retención aguda de orina suele aparecer en el postoperatorio inmediato, o a la largo de las primeras semanas, en un momento en el que todavía no se ha liberado la mayor parte de la irradiación. Por ello es más plausible que el mecanismo de producción de la retención urinaria sea el traumatismo directo sobre la próstata asociado a la inserción de las agujas. En esta línea es interesante el trabajo de Buskirk et al. (40), analizando la incidencia de retención aguda de orina tras biopsia prostática transperineal. Observan una incidencia de RAO del $11.5 \%$, similar a las series de braquiterapia prostática, relacionando la retención con el volumen prostático y el número de cilindros de biopsia. En este sentido, Lee et al. también observan correlación entre la incidencia de RAO y el número de agujas insertadas (41).

Casi todos los trabajos coinciden al asociar la mayor incidencia de RAO en pacientes con mayores volúmenes prostáticos. Especialmente, aquellas próstatas con adenomas más grandes (mayor volumen de zona transicional, esto es, un índice de zona transicional mayor) tienen mayor incidencia de RAO, como lo demuestran algunos autores $(42,43)$. El edema y sangrado en la zona transicional asociado al paso de las agujas serían los responsables del aumento de resistencia uretral que condicionaría finalmente retención urinaria.

Se han postulado varios factores predictivos de RAO, entre ellos la flujometría, residuo postmiccional, puntuación IPSS, bloqueo hormonal pretratamiento, etc. De todos ellos, el más coincidente en diversas publicaciones es una alta puntuación IPSS pretratamiento $(28-30,37,38)$. 
En resumen, la RAO ocurre habitualmente al día siguiente del implante y probablemente está relacionada con el edema postimplante y los efectos de los agentes anestésicos, ya que la radiación liberada es todavía insignificante. Pese a todo ello, el índice de retenciones urinarias es bajo, y se suele resolver con la colocación de una sonda vesical por espacio de unas semanas y tratamiento médico con $\alpha$-bloqueantes. Pocos de estos pacientes requerirán una resección transuretral de próstata (RTUP) desobstructiva por RAO refractaria.

\section{RESECCIÓN TRANSURETRAL POSTBRAQUITE- RAPIA}

Algunos pacientes que desarrollan RAO mantenida o síntomas obstructivos severos pueden requerir RTU prostática postimplante. Las tasas de RTUP post implante oscilan del 0 al $8 \%(12,16,28,33$, 44) (Tabla I). En caso de tener que llevar a cabo una RTUP hay que esperar un tiempo mínimo de seguridad después del implante, unos seis meses para el 1125 y dos meses para el Pd103, tiempo en el que el $90 \%$ de la radiación ha sido emitida. La RTUP, a fin de evitar la incontinencia urinaria post-implante, debe ser cuidadosa, comenzando la resección por el lóbulo medio y posteriormente resecando los lóbulos laterales. Se debe intentar conservar el cuello vesical a las 5 y las $7 \mathrm{~h}$ para preservar la principal irrigación de la uretra prostática. La resección del tejido de los lóbulos laterales debe ser mínima, teniendo extremo cuidado con la resección del ápex, quitando una cantidad mínima de tejido sólo si es necesario, ya que podría estar ya comprometido por la exposición a la radiación previa $(27,45)$. Mediante la RTUP se obtendrán una media por resección de 5 a 10 semillas del interior de la próstata. Cuando una semilla es expuesta en la resección o el movimiento de corte con el asa de resección nota resistencia, se debe realizar un corte más profundo recolocando el asa. Esta maniobra facilita la retirada posterior de las semillas con el evacuador Ellik. Las semillas deben ser recogidas y entregadas en la oficina de seguridad de radiación para una eliminación adecuada. Posteriormente debe dejarse una sonda de 20-22 Fr durante una media de 2 días (45). La RTUP no debe ser inmediata a la retención para diferenciar los pacientes con una retención urinaria refractaria de los que presentan una retención prolongada, en los que el mantenimiento por un tiempo prolongado de la sonda vesical resolverá el cuadro (28).

TABLA I. COMPLICACIONES URINARIAS DE LA BRAQUITERAPIA PROSTÁTICA.

\begin{tabular}{|c|c|c|c|}
\hline & RAO $\mathbf{n}(\%)$ & RTU-P $\mathbf{n}(\%)$ & Incontinencia Urinaria \\
\hline Prada (2005) (2) & $(3)$ & $(0)$ & $(0)$ \\
\hline Aguiló (2005) (60) & $6(4,35)$ & - & - \\
\hline Wallner (1996) (33) & $13(14)$ & $8(8,7)$ & - \\
\hline Terk (1998) (28) & $14(5,5)$ & $6(2,4)$ & $95(6,7)$ \\
\hline Benoit (2000) (61) & $205(14,5)$ & $122(8,7)$ & $4(0,7)$ \\
\hline Gelblum (1999) (12) & - & $28(4,7)$ & - \\
\hline Sarosdy (2004) (31) & $24(24)$ & $5(5,2)$ & $0(0)$ \\
\hline Merrick (2000) (16) & $10(6)$ & $2(1,2)$ & $1(0,7)$ \\
\hline Guinot (2005) (62) & $9(7)$ & $2(1,5)$ & $1(1,04)$ \\
\hline Tormo (2006)^ & $6(6,25)$ & $2(2,08)$ & \\
\hline
\end{tabular}

$\wedge$ Datos no publicados 
Las tasas de incontinencia post-RTUP tras braquiterapia oscilan entre el $18-70 \%$ según las series publicadas (Tabla II). El tiempo óptimo de espera para una RTUP tras tratamiento con radiación es controvertido. Así, estudios realizados en pacientes tratados con radioterapia externa sugieren que la tasa de incontinencia post RTUP aumenta cuanto menor sea el tiempo transcurrido desde la radiación (46). Sin embargo, Kollmeier et al., observaron tasas de incontinencia mayores en pacientes tratados con braquiterapia y RTUP posterior, cuando ésta se realizaba a partir de los dos años postimplante. Los pacientes con retención secundaria al edema agudo del implante tenían menor probabilidad de retención tras una RTUP que aquellos que presentaban síntomas obstructivos tardíos (tras 2 años) probablemente secundarios a la fibrosis inducida por la irradiación (45). Sin embargo la principal limitación de este estudio es su corta muestra (38 pacientes con RTUP), por lo que son necesarios más estudios, con mayor número de pacientes, que permitan aclarar el tiempo óptimo de espera previo a una RTUP.

\section{INCONTINENCIA}

La incontinencia urinaria es probablemente el síntoma urinario más temido y que más afecta la calidad de vida del paciente. Su incidencia es muy variable, entre el 0 y el $83 \%$ según las series consultadas, y esta amplia variabilidad depende de la definición de incontinencia, del momento de evaluación, y del procedimiento efectuado. Así, la incontinencia urinaria como complicación crónicamente establecida, es rara, siendo mucho mayor (llegando al $83 \%$ en alguna serie) en pacientes sometidos a RTUP previa (47), como ya hemos comentado. En estos casos, la incontinencia se produce por sobreirradiación de una uretra prostática ya operada, y de algún modo puede prevenirse si se efectúa una carga periférica. Por otro lado, aquellos pacientes que necesitan una RTUP postimplante por problemas obstructivos, tienen un mayor riesgo de incontinencia, por un doble mecanismo: la irradiación uretral y el daño térmico de la RTUP, que provocan una hipovascularización, necrosis superficial y posible fibrosis de uretra prostática y membranosa (48). La fibrosis uretral y esfinteriana hace muy difícil la resolución quirúrgica de esta incontinencia.

De forma clásica, los pacientes con antecedentes de RTUP no eran candidatos a braquiterapia, por un doble motivo: el riesgo claramente aumentado de incontinencia postimplante y la dificultad técnica de ubicar correctamente las semillas en la zona periférica, dada la cavidad creada a nivel de uretra prostática. Una carga periférica de las semillas, cuidadosamente ubicadas para minimizar la dosis uretral, puede solventar este problema en algunos pacientes, aunque es necesario advertir del mayor riesgo de complicaciones urinarias.

\section{FACTORES PREDICTIVOS DE COMPLICACIONES URINARIAS}

Como ya se ha comentado en apartados anteriores, de los diversos trabajos publicados se concluye que algunos factores están claramente relacionados con la aparición de complicaciones urinarias, como pueden ser el volumen prostático o las altas puntuaciones en el cuestionario IPSS preimplante. Otros factores no son tan unánimes, y resultan incluso contradictorios según la experiencia de los distintos autores.

TABLA II. INCONTINENCIA URINARIA POST RTU-P EN PACIENTES TRATADOS CON BRAQUITERAPIA PROSTÁTICA.

\begin{tabular}{|c|c|c|c|c|}
\hline & $\mathbf{n}$ & Tratamiento & Incontinencia n (\%) & Correlación \\
\hline $\mathrm{Hu}(63)$ & 10 & Implante I25 & $7(70)$ & Dosis máx uretral > 400 Gy \\
\hline Gelblum (12) & 28 & Implante $\pm \mathrm{RT}$ & $5(17)$ & ninguna \\
\hline Koutrouvelis (64) & 11 & Implante I125 $\circ \mathrm{Pd}^{103}$ & $3(27)$ & ninguna \\
\hline Kollmeier (45) & 38 & Implante I125 $\circ \mathrm{Pd}^{103} \pm \mathrm{RT}$ & $7(18)$ & ninguna \\
\hline
\end{tabular}

RT: radioterapia externa 
Casi todos los autores coinciden en considerar el volumen prostático preimplante como predictivo de RAO $(12,14,17,19,22,29,37-39)$. Gelblum et al. (12) observaron mayor riesgo de síntomas urinarios y retención en pacientes con próstatas $>35$ cc. Thomas et al. (43) en un análisis multivariado, afinan más y eliminan el volumen prostático global como factor independiente, y lo sustituyen por el índice de zona transicional. La importancia del volumen prostático estriba no sólo en que es un factor predictivo de complicaciones urinarias (especialmente la RAO), sino en que las próstatas de gran tamaño dificultan incluso impiden una adecuada siembra de semillas radioactivas en su parénquima, al ofrecer el arco púbico un límite anatómico a veces infranqueable. Es por ello que algunos pacientes precisan de un bloqueo hormonal previo que haga disminuir el tamaño prostático hasta un límite técnicamente factible para la braquiterapia (habitualmente $<50 \mathrm{cc}$ ). Por otra parte, se ha observado una asociación entre retención urinaria y necesidad de sondaje vesical postbraquiterapia y los cambios en la anatomía prostática inducidos por bloqueo hormonal (cambios en la disposición uretral asociados a la disminución de tamaño de la zona transicional) (24).

Otro factor anatómico prostático clásicamente considerado es la presencia de un lóbulo medio, por su tendencia a crear problemas obstructivos. Se ha postulado la necesidad de efectuar RTUP del lóbulo medio exclusivamente antes de indicar un implante. Sin embargo, Wallner et al. (49) en una serie corta de 8 pacientes con lóbulo medio sometidos a braquiterapia prostática observaron dos retenciones agudas de orina, precisando un paciente cateterismo limpio intermitente durante 3 meses. Concluyen que no es una contraindicación absoluta y que no sería necesaria la RTU profiláctica del lóbulo medio.

Puntuaciones altas del IPSS pretratamiento parecen estar asociadas con la aparición de RAO. Así, según la experiencia de Terk et al. (28), el único factor predictivo que alcanzaba significación estadística en el análisis multivariante era el IPSS, de forma que los pacientes con IPSS $<10$ tenían una incidencia de retención urinaria del $2 \%$, con IPSS 10 19 del $11 \%$ y con IPSS>19 del 29\%. Esta idea de que a mayor puntuación IPSS pretratamiento, mayor probabilidad de retención urinaria ha sido abalada por otros autores $(29,30,37,38)$, aunque no todos han encontrado esta asociación en sus estudios. Esta discordancia se podría explicar porque precisamente, los pacientes con mayores puntuaciones sintomáticas en el IPSS no suelen incluirse habitualmente en los protocolos de braquiterapia prostática. De este modo, es difícil encontrar asociaciones estadísticas en los estudios multivariantes. Por otro lado, el IPSS cubre un espectro de síntomas de llenado y vaciado, siendo únicamente los de vaciado los que se asocian con más probabilidad al proceso obstructivo. Así, Kelly et al. (13) observaron que el chorro débil del cuestionario IPSS era el único ítem que se asociaba de forma estadísticamente significativa en el análisis de regresión logística al riesgo de cateterismo prolongado.

Además de los puramente anatómicos (volumen prostático, lóbulo medio) y clínicos (IPSS), los factores funcionales también han de tenerse en cuenta a la hora de predecir posibles complicaciones urinarias. Sin embargo, pocos son los grupos de trabajo que han tenido en cuenta estos factores en sus estudios. Henderson et al. (38) observaron que el estado funcional urodinámico pretratamiento era una variable predictiva independiente de retención urinaria en el análisis multivariante. El flujo máximo pretratamiento se ha postulado como factor predictivo independiente de retención, aunque sin poder establecer un punto de corte diferencial (18). Beekman et al. (50) concluyen en un estudio multivariante sobre 204 pacientes implantados, que la selección de pacientes con residuo postmiccional $<100 \mathrm{ml}$ evita la aparición de RAO prolongada (más de 3 días), evita la necesidad de RTUP postimplante y se asocia a una más rápida normalización del IPSS. Wehle et al. (51) establecen en su serie, un grupo de alto riesgo de complicaciones urinarias (presencia de uno o más de los siguientes factores): IPSS > 15, Qmax < $10 \mathrm{ml} / \mathrm{s}$, residuo $>100 \mathrm{ml}$ y volumen prostático $>40$ cc. Observan un $37 \%$ de complicaciones urinarias en este grupo frente a un $15 \%$ del grupo de bajo riesgo ( $p=0.006)$, siendo el Qmax el factor individual más predictivo de complicaciones.

Las Guías de La Asociación Europea de Urología no son muy específicas a la hora de recomendar criterios clínicos o funcionales de pacientes candidatos a braquiterapia, primando como es de esperar los criterios oncológicos sobre los funcionales (52). De los factores que se han comentado anteriormente, incluye como pacientes candidatos a braquiterapia a aquellos con próstatas $<50 \mathrm{cc}$ y con una buena puntuación sintomática IPSS (53). No establece ningún punto de corte idóneo para la puntuación IPSS, ni incluye criterios funcionales como la flujometría, residuo postmiccional, o parámetros urodinámicos de obstrucción. Queda a criterio de cada grupo de trabajo el establecimiento de puntos de corte propios para la inclusión o no de un paciente concreto. Parece lógico que el candidato idóneo desde un punto de vista funcional debería tener un IPSS previo $<15$, Qmax $>10 \mathrm{ml} / \mathrm{s}$, residuo postmiccional $<100 \mathrm{ml}$ y sin signos de obstrucción en el estudio de presión y flujo (número de Abrams y Griffith < 40). 


\section{MEDIDAS PROFILÁCTICAS}

Probablemente la mejor medida profiláctica que se puede tomar para evitar las complicaciones urinarias de la braquiterapia prostática es efectuar una adecuada selección de pacientes.

Wein, en un comentario editorial al trabajo de Blaivas et al. (26), critica la alta incidencia de efectos secundarios urinarios de este grupo y atribuye la baja incidencia de efectos secundarios urinarios crónicos en su propio medio de trabajo a varios factores de selección:

1) descartan a pacientes con próstatas grandes o con lóbulos medios obstructivos;

2) descartan pacientes con STUI severos prebraquiterapia;

3) evalúan cistoscópicamente a todos los pacientes;

4) efectúan estudio urodinámico en todos los pacientes con STUI sugestivos de obstrucción prostática;

5) descartan a todos los pacientes con datos sugestivos de obstrucción.

Desde un punto de vista farmacológico se han utilizado alfabloqueantes, corticoides y bloqueo hormonal, para minimizar la incidencia de complicaciones urinarias, con resultados diversos.

Los alfabloqueantes se han utilizado de forma terapéutica para tratar los síntomas urinarios postimplante, y también de forma profiláctica para prevenir su aparición. Es muy demostrativo el trabajo de Merrick et al. (32), en el que dividen dos grupos de pacientes implantados: a los primeros se les administra alfabloqueante de forma profiláctica (2 semanas antes del implante y mantenido posteriormente) y a los segundos o no se les trata o se les da el alfabloqueante cuando aparecen los síntomas urinarios. Aunque la asignación no fue aleatoria, se observaron diferencias significativas entre los grupos: los síntomas se normalizaron más rápidamente en el grupo profiláctico (3 meses vs 6) y la puntuación IPSS se elevó menos en el grupo profiláctico (4 vs 11 ). Sin embargo, el uso de un alfabloqueante no parece reducir el riesgo de retención urinaria prolongada (más de 3 días) ni reduce la necesidad de cirugía desobstructiva postimplante (15).

Es significativo un estudio doble ciego, aleatorizado y controlado con placebo efectuado con $0.8 \mathrm{mg}$ de tamsulosina administrada 4 días antes y 60 días postimplante. No observan diferencias en la incidencia de RAO entre el grupo placebo (17\%) y con tamsulosina (10\%), ni tampoco en la puntuación sintomática a las dos semanas del implante. Sin embargo, sí observan diferencias en la puntuación IPSS a las 5 semanas a favor de tamsulosina (54). Así pues, serían útiles en mejorar la sintomatología a medio plazo, no en el periodo inmediato postimplante.

Se ha propuesto el uso de corticoides durante y posteriormente al implante, con la idea de disminuir el edema, mejorar los síntomas y disminuir el riesgo de retención urinaria (55). Sin embargo otros estudios no han conseguido demostrar diferencias significativas entre usar o no esteroides $(56,57)$.

El bloqueo hormonal previo a braquiterapia prostática tiene su indicación principal en próstatas de gran tamaño, para conseguir una reducción de volumen que las haga adecuadas al implante (58). Un trabajo de Blank et al. (59) compara pacientes con bloqueo hormonal 3 meses preimplante con pacientes sin bloqueo, y observan un menor volumen prostático (27.7 cc vs $36.3 \mathrm{cc})$, menor uso de semillas (47.9 vs 83.2 ) y menos dosis de radiación $(76.3$ $\mathrm{mCi}$ vs $117 \mathrm{mCi}$ ), lo que se tradujo en menor morbilidad genitourinaria (59).

\section{CONCLUSIONES}

La aparición de síntomas urinarios postbraquiterapia es un hecho muy frecuente, que alcanza su máximo entre el primer y tercer mes, normalizándose alrededor del año. Un porcentaje bajo de pacientes necesitará una cirugía desobstructiva para resolver una retención urinaria prolongada o síntomas obstructivos severos. Los alfabloqueantes pueden ser útiles para aliviar los síntomas urinarios postimplante.

Existen varios factores clínicos predictivos de aparición de complicaciones urinarias. Un paciente con puntuación IPSS preimplante alta, próstata de gran tamaño, con signos funcionales obstructivos (Qmax bajo, residuo alto y parámetros urodinámicos de obstrucción), tiene alto riesgo de presentar retención urinaria o síntomas urinarios prolongados. Esto repercutirá claramente en la calidad de vida del paciente, lo que lo hace candidato a otro tipo de terapias.

El empleo de una técnica de implantación periférica (que asegure bajas dosis en uretra) y evitar el plano uretral en la inserción de las agujas puede minimizar el daño uretral asociado a la aparición de complicaciones urinarias. 
La incontinencia postbraquiterapia es una complicación poco frecuente, pero que aumenta de forma dramática en pacientes con RTUP previa o que precisan de la misma para resolver una retención urinaria mantenida. Es fundamental conocer esta circunstancia para informar debidamente al paciente y planificar una RTUP lo más cuidadosa posible.

\section{BIBLIOGRAFÍA Y LECTURAS RECOMENDADAS ( ${ }^{*}$ lectura de interés $y^{* *}$ lectura fundamental)}

1. Potters L, Klein E A, Kattan M W, et al. Monotherapy for stage T1-T2 prostate cancer: radical prostatectomy, external beam radiotherapy, or permanent seed implantation. Radiother Oncol, 2004; 71: 29.

*2. Prada P J, Hevia M, Juan G, et al. I125 low dose rate brachytherapy in localized prostate cancer. Preliminary results after 5 years. Arch Esp Urol, 2005; 58: 213.

3. Mabjeesh N, Chen J, Beri A, et al. Sexual function after permanent 125I-brachytherapy for prostate cancer. Int J Impot Res, 2005; 17: 96.

4. Robinson J, Moritz S, Fung T. Meta-analysis of rates of erectile function after treatment of localized prostate carcinoma. Int J Radiat Oncol Biol Phys, 2002; 54: 1063.

*5. Mallick S, Azzouzi R, Cormier L, et al. Urinary morbidity after $125 \mathrm{I}$ brachytherapy of the prostate. BJU Int, 2003; 92: 555.

6. Quimby E H. The grouping of radio tubes in packs and plaques to produce the desired distribution of radiation. Am J Roentgenol, 1932; 18.

7. Paterson R, Parker H M. A dosage system for gamma-ray therapy. Br J Radiol, 1943; 592.

8. Stone N N, Stock R G. Brachytherapy for prostate cancer: real-time three-dimensional interactive seed implantation. Tech Urol, 1995; 1: 72

9. Wallner K, Roy J, Harrison L. Dosimetry guidelines to minimize urethral and rectal morbidity following transperineal I-125 prostate brachytherapy. Int J Radiat Oncol Biol Phys, 1995; 32: 465.

10. Salem N. [Clinical and biological surveillance after radiotherapy for localized prostate cancer]. Cancer Radiother, 2002; 6: 159.

11. Wallner K, Merrick G, True L, et al. I-125 versus Pd-103 for low-risk prostate cancer: morbidity outcomes from a prospective randomized multicenter trial. Cancer J, 2002; 8: 67.

12. Gelblum D Y, Potters L, Ashley R, et al. Urinary morbidity following ultrasound-guided transperineal prostate seed implantation. Int J Radiat Oncol Biol Phys, 1999; 45: 59.

13. Kelly K, Swindell R, Routledge J, et al. Prediction of urinary symptoms after 125 iodine prostate brachytherapy. Clin Oncol (R Coll Radiol), 2006; 18: 326.

**14. Merrick G S, Wallner K, Butler W M. Minimizing prostate brachytherapy-related morbidity. Urology, 2003; 62: 786.

15. Desai J, Stock R G, Stone N N et al. Acute urinary morbidity following I-125 interstitial implantation of the prostate gland. Radiat Oncol Investig, 1998; 6: 135

16. Merrick G S, Butler W M, Lief J H et al. Temporal resolution of urinary morbidity following prostate brachytherapy. Int J Radiat Oncol Biol Phys, 47: 121,2000

**17. Steggerda, M. J., van der Poel, H. G., Moonen, L. M.: An analysis of the relation between physical characteristics of prostate I-125 seed implants and lower urinary tract symptoms: bladder hotspot dose and prostate size are significant predictors. Radiother Oncol, 2008; 88: 108.

18. Williams S G, Millar J L, Duchesne G M et al. Factors predicting for urinary morbidity following 125iodine transperineal prostate brachytherapy. Radiother Oncol, 2004; 73: 33.

19. Salem N, Simonian-Sauve M, Rosello R, et al. Predictive factors of acute urinary morbidity after iodine-125 brachytherapy for localised prostate cancer: a phase 2 study. Radiother Oncol, 2003; 66: 159 .

20. Van Gellekom M P, Moerland M A, Van Vulpen $\mathrm{M}$, et al. Quality of life of patients after permanent prostate brachytherapy in relation to dosimetry. Int J Radiat Oncol Biol Phys, 2005; 63: 772.

21. Wust P, Von Borczyskowski D W, Henkel T, et al. Clinical and physical determinants for toxicity of 125-I seed prostate brachytherapy. Radiother Oncol, 2004; 73: 39.

22. Gutman S, Merrick G S, Butler W M, et al. Severity categories of the International Prostate Symptom Score before, and urinary morbidity after, permanent prostate brachytherapy. BJU Int, 2006; 97: 62.

23. Niehaus A, Merrick G S, Butler W M, et al. The influence of isotope and prostate volume on urinary morbidity after prostate brachytherapy. Int $\mathbf{J}$ Radiat Oncol Biol Phys, 2006; 64: 136.

24. Hinerman-Mulroy A, Merrick G S, Butler W M, et al. Androgen deprivation-induced changes in prostate anatomy predict urinary morbidity after permanent interstitial brachytherapy. Int J Radiat Oncol Biol Phys, 2004; 59: 1367.

25. Allen Z A, Merrick G, Butler E B. Detailed urethral dosimetry in the evaluation of prostate brachytherapy related urinary morbidity. Int J Radiat Oncol Biol Phys, 2005; 62: 453

*26. Blaivas J G, Weiss J P, Jones M. The pathophysiology of lower urinary tract symptoms after brachytherapy for prostate cancer. BJU Int, 2006; 98: 1233. 
**27. Stone N N, Stock R G. Complications following permanent prostate brachytherapy. Eur Urol, 2002; 41: 427

28. Terk M D, Stock R G, Stone N N. Identification of patients at increased risk for prolonged urinary retention following radioactive seed implantation of the prostate. J Urol, 1998; 160: 1379.

29. Crook J, McLean M, Catton C, et al. Factors influencing risk of acute urinary retention after TRUS-guided permanent prostate seed implantation. Int J Radiat Oncol Biol Phys, 2002; 52: 453.

30. Locke J, Ellis W, Wallner K, et al. Risk factors for acute urinary retention requiring temporary intermittent catheterization after prostate brachytherapy: a prospective study. Int J Radiat Oncol Biol Phys, 2002; 52: 712.

31. Sarosdy M F. Urinary and rectal complications of contemporary permanent transperineal brachytherapy for prostate carcinoma with or without external beam radiation therapy. Cancer, 2004; 101: 754.

32. Merrick G S, Butler W M, Wallner K E et al. Prophylactic versus therapeutic alpha-blockers after permanent prostate brachytherapy. Urology, 2002; 60: 650.

33. Wallner K, Roy J, Harrison L. Tumor control and morbidity following transperineal iodine $125 \mathrm{im}$ plantation for stage $\mathrm{T} 1 / \mathrm{T} 2$ prostatic carcinoma. $\mathrm{J}$ Clin Oncol, 1996; 14: 449.

34. Mallick S, Azzouzi R, Cormier L, et al. Urinary morbidity after $125 \mathrm{I}$ brachytherapy of the prostate. BJU, 2003; 92: 555.

35. Prada P, Hevia M, Juan G, et al. Braquiterapia de baja tasa (I125) en el cáncer de prostata localizado. Arch Esp Urol, 2005; 58: 213.

36. Bottomley D, Ash D, Al-Qaisieh B, et al. Side effects of permanent I125 prostate seed implants in 667 patients treated in Leeds. Radiother Oncol, 2007; 82: 46.

37. Bucci J, Morris W J, Keyes M, et al. Predictive factors of urinary retention following prostate brachytherapy. Int J Radiat Oncol Biol Phys, 2002; 53: 91.

*38. Henderson A, Ismail A K, Cunningham M, et al. Toxicity and early biochemical outcomes from 125iodine prostate brachytherapy in the UK. A prospective study. Clin Oncol (R Coll Radiol), 2004; 16: 95.

39. Martin A G, Roy J, Beaulieu L, et al. Permanent prostate implant using high activity seeds and inverse planning with fast simulated annealing algorithm: A 12-year Canadian experience. Int J Radiat Oncol Biol Phys, 2007; 67: 334.

40. Buskirk S J, Pinkstaff D M, Petrou S P, et al. Acute urinary retention after transperineal templateguided prostate biopsy. Int $\mathbf{J}$ Radiat Oncol Biol Phys, 2004; 59: 1360.
41. Lee N, Wuu C S, Brody R, et al. Factors predicting for postimplantation urinary retention after permanent prostate brachytherapy. Int J Radiat Oncol Biol Phys, 2000; 48: 1457.

42. Kaplan S A, Te A E, Pressler L B, et al. Transition zone index as a method of assessing benign prostatic hyperplasia: correlation with symptoms, urine flow and detrusor pressure. J Urol, 1995; 154: 1764.

43. Thomas M D, Cormack R, Tempany C M, et al. Identifying the predictors of acute urinary retention following magnetic-resonance-guided prostate brachytherapy. Int J Radiat Oncol Biol Phys, 2000;47: 905

44. Storey M R, Landgren R C, Cottone J L, et al.Transperineal 125iodine implantation for treatment of clinically localized prostate cancer: 5 -year tumor control and morbidity. Int J Radiat Oncol Biol Phys, 1999; 43: 565.

45. Kollmeier M A, Stock R G, Cesaretti J, et al. Urinary morbidity and incontinence following transurethral resection of the prostate after brachytherapy. J Urol, 2005; 173: 808.

46. Hirshberg E D, Klotz L H. Post transurethral resection of prostate incontinence in previously radiated prostate cancer patients. Can J Urol, 1998; 5: 560 .

47. Talcott J A, Clark J A, Stark P C, et al. Long-term treatment related complications of brachytherapy for early prostate cancer: a survey of patients previously treated. J Urol, 2001; 166: 494.

48. Ragde H, Elgamal A A, Snow P B, et al. Ten-year disease free survival after transperineal sonography-guided iodine-125 brachytherapy with or without 45-gray external beam irradiation in the treatment of patients with clinically localized, low to high Gleason grade prostate carcinoma. Cancer, 1998; 83: 989.

49. Wallner K, Smathers S, Sutlief S, et al. Prostate brachytherapy in patients with median lobe hyperplasia. Int J Cancer, 2000; 90: 152.

50. Beekman M, Merrick G S, Butler W M, et al. Selecting patients with pretreatment postvoid residual urine volume less than $100 \mathrm{~mL}$ may favorably influence brachytherapy-related urinary morbidity. Urology, 2005; 66: 1266.

51. Wehle M J, Lisson S W, Buskirk S J, et al. Prediction of genitourinary tract morbidity after brachytherapy for prostate adenocarcinoma. Mayo Clin Proc, 2004; 79: 314.

*52. Heidenreich A, Bolla M, Joniau S, et al. Guidelines on Prostate Cancer. In: EAU Guidelines. Edited by E. A. o. Urology. Arnhem, 2009.

*53. Ash D, Flynn A, Battermann J, et al. ESTRO/ EAU/EORTC recommendations on permanent seed implantation for localized prostate cancer. Radiother Oncol, 2000; 57: 315. 
54. Elshaikh M A, Ulchaker J C, Reddy C A, et al. Prophylactic tamsulosin (Flomax) in patients undergoing prostate $125 \mathrm{I}$ brachytherapy for prostate carcinoma: final report of a double-blind placebocontrolled randomized study. Int J Radiat Oncol Biol Phys, 2005; 62: 164.

55. Sacco D E, Daller M, Grocela J A, et al. Corticosteroid use after prostate brachytherapy reduces the risk of acute urinary retention. BJU Int, 2003; 91: 345 .

56. Merrick G S, Butler W M, Dorsey A T, et al. Influence of prophylactic dexamethasone on edema following prostate brachytherapy. Tech Urol, 2000; 6: 117.

57. Mierzwa M L, Barrett W L, Redmond K, et al. Randomized trial to assess the efficacy of intraoperative steroid use in decreasing acute urinary retention after transperineal radioactive iodine125 implantation for prostate cancer. Cancer, 2008.

58. Kucway R, Vicini F, Huang R, et al. Prostate volume reduction with androgen deprivation therapy before interstitial brachytherapy. J Urol, 2002; 167: 2443.
59. Blank K R, Whittington R, Arjomandy B, et al.Neoadjuvant androgen deprivation prior to transperineal prostate brachytherapy: smaller volumes, less morbidity. Cancer J Sci Am, 1999; 5: 370.

60. Aguilo Lucia F, Suarez Novo J F, Correa Generoso R, et al. [Retrospective study of 130 patients with organconfined prostate cancer treated with brachytherapy]. Actas Urol Esp, 2005; 29: 47.

61. Benoit R M, Naslund M J, Cohen J K. Complications after prostate brachytherapy in the Medicare population. Urology, 2000; 55: 91.

62. Guinot J L, Estornell R, Escolar P P. What have we learned in the first two years about I-125 prostate brachytherapy?. Edited by ESTROCongress. Budapest: Radiother Oncol 75 supl (2005) S-36, 2005.

63. Hu K, Wallner K. Urinary incontinence in patients who have a TURP/TUIP following prostate brachytherapy. Int J Radiat Oncol Biol Phys, 1998; 40: 783 .

64. Koutrouvelis P G, Lailas N, Katz S, et al. Prostate cancer with large glands treated with 3-dimensional computerized tomography guided pararectal brachytherapy: up to 8 years of followup. J Urol, 2003; 169: 1331. 